\title{
Effect of Imperfections and Damping on the Type of Nonlinearity of Circular Plates and Shallow Spherical Shells
}

\author{
Cyril Touzé, ${ }^{1}$ Cédric Camier, ${ }^{1}$ Gaël Favraud, ${ }^{1}$ and Olivier Thomas ${ }^{2}$ \\ ${ }^{1}$ ENSTA-UME, Unité de Mécanique, Chemin de la Hunière, 91761 Palaiseau Cedex, France \\ ${ }^{2}$ CNAM-LMSSC, Laboratoire de Mécanique des Structures et Systèmes Couplés, \\ 2 rue Conté, 75003 Paris, France
}

Correspondence should be addressed to Cyril Touzé, cyril.touze@ensta.fr

Received 28 November 2007; Accepted 20 February 2008

Recommended by Paulo Gonçalves

The effect of geometric imperfections and viscous damping on the type of nonlinearity (i.e., the hardening or softening behaviour) of circular plates and shallow spherical shells with free edge is here investigated. The Von Kármán large-deflection theory is used to derive the continuous models. Then, nonlinear normal modes (NNMs) are used for predicting with accuracy the coefficient, the sign of which determines the hardening or softening behaviour of the structure. The effect of geometric imperfections, unavoidable in real systems, is studied by adding a static initial component in the deflection of a circular plate. Axisymmetric as well as asymmetric imperfections are investigated, and their effect on the type of nonlinearity of the modes of an imperfect plate is documented. Transitions from hardening to softening behaviour are predicted quantitatively for imperfections having the shapes of eigenmodes of a perfect plate. The role of 2:1 internal resonance in this process is underlined. When damping is included in the calculation, it is found that the softening behaviour is generally favoured, but its effect remains limited.

Copyright @ 2008 Cyril Touzé et al. This is an open access article distributed under the Creative Commons Attribution License, which permits unrestricted use, distribution, and reproduction in any medium, provided the original work is properly cited.

\section{Introduction}

When continuous structures such as plates and shells undergo large amplitude motions, the geometrical nonlinearity leads to a dependence of free oscillation frequencies on vibration amplitude. The type of nonlinearity describes this dependency, which can be of the hardening type (the frequency increases with amplitude), or of the softening type (the frequency decreases). A large amount of literature is devoted to predicting this type of nonlinearity for continuous structures, and especially for structures with an initial curvature such as arches or shells because the presence of the quadratic nonlinearity makes the problem more difficult to solve. On the other hand, the hardening behaviour of flat structures such as beams and plates 
is a clearly established fact, on the theoretical as well as the experimental viewpoint, (see, e.g., [1-6]). The presence of the quadratic nonlinearity may change the behaviour from hardening to softening type, depending on the relative magnitude of quadratic and cubic nonlinear terms.

Among the available studies concerned with this subject, quite all of them that were published before 1992 could not be considered as definitive since they generally restrict to the case of a single-mode vibration through Galerkin method, see, for example, [7-9] for shallow spherical shells, or [10] for imperfect circular plates. Unfortunately, it has been shown by a number of more recent investigations that too severe truncations lead to erroneous results in the prediction of the type of nonlinearity, see, for example, [11, 12], or the abundant literature on circular cylindrical shells, where the investigators faced this problem for a long time [1318]. As a consequence, a large number of modes must mandatory be kept in the truncation of the partial differential equations (PDEs) of motion, in order to accurately predict the type of nonlinearity. Recent papers are now available where a reliable prediction is realized, for the case of buckled beams [19], circular cylindrical shells [20], suspended cables [21], and shallow spherical shells [22].

However, these last studies are restricted to the case of perfect structures, and the damping is neglected in the computations; and both of them have an influence on the type of nonlinearity, so that a complete and thorough theoretical study that could be applied to real structures need to address the effect of imperfections and damping. The geometric imperfections have a first-order effect on the linear as well as the nonlinear characteristics of structures. A large amount of studies are available, where the effect of imperfections on the eigenfrequencies and on the buckling loads are generally addressed, see, for example, [2328] for the case of circular cylindrical shells, [29] for shallow cylindrical panels, and [30] for the case of rectangular plates. Nonlinear frequency-responses curves are shown in $[31,32]$ for clamped circular plates, [33-35] for rectangular plates, [36] for circular cylindrical shells, and [37] for circular cylindrical panels. Even though the presence of geometric imperfection has been recognized as a major factor that could make the hardening behaviour of the flat plate turn to softening behaviour for an imperfection amplitude of a fraction of the plate thickness $[10,38]$, a quantitative study, which is not restricted to axisymmetric modes and that does not perform too crude truncations in the Galerkin expansion, is still missing.

To the authors' knowledge, the role of the damping in the prediction of the type of nonlinearity has been only recently detected as an important factor that could change the behaviour from hardening to softening type [39]. In particular, it is shown in [39] on a simple two degrees-of-freedom (dofs) system, that the damping generally favours the softening behaviour. The aim of the present study is thus to apply this theoretical result to the practical case of a damped shallow spherical shell, so as to quantitatively assess the effect of structural damping of the viscous type on the type of nonlinearity of a two-dimensional vibrating structure.

The article is organized as follows. In Section 2, local equations and boundary conditions for an imperfect circular plate with free edge are given. Then the method used for computing the type of nonlinearity is explained. Section 3 investigates how typical imperfections may turn the hardening behaviour of flat plates to softening behaviour. Quantitative results are given for selected imperfections having the shape of eigenmodes of the perfect structure. Section 4 is devoted to the effect of viscous damping. The particular case of a spherical imperfection is selected, and the results are shown for three different damping dependances on frequency. 


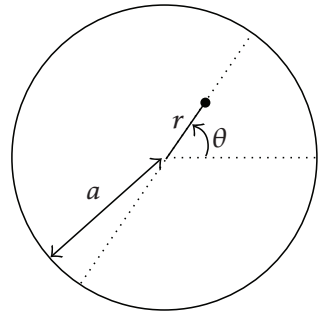

(a)

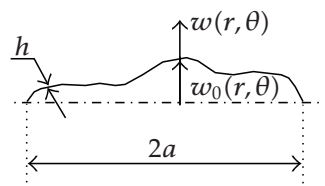

(b)

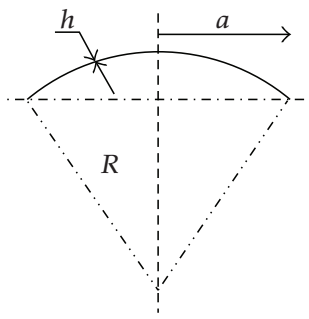

(c)

Figure 1: (a) Top view and (b) cross-section of an imperfect circular plate of radius $a$ and thickness $h$. (c) The particular case of a spherical imperfection, with radius of curvature $R$.

\section{Theoretical formulation}

\subsection{Local equations and boundary conditions}

A thin plate of diameter $2 a$ and uniform thickness $h$ is considered, with $h \ll a$, and freeedge boundary condition. The local equations governing the large-amplitude displacement of a perfect plate, assuming the nonlinear Von Kármán strain-displacement relationship and neglecting in-plane inertia, are given, for example, in [5, 40]. An initial imperfection, denoted by $w_{0}(r, \theta)$ and associated with zero inital stresses is also considered, see Figure 1 . The shape of this imperfection is arbitrary, and its amplitude is small compared to the diameter (shallow assumption): $w_{0}(r, \theta) \ll a$. The local equations for an imperfect plate deduce from the perfect case $[18,41,42]$. With $w(r, \theta, t)$ being the transverse displacement from the imperfect position at rest, the equations of motion write

$$
\begin{gathered}
D \Delta \Delta w+\rho h \ddot{w}=L(w, F)+L\left(w_{0}, F\right)-c \dot{w}, \\
\Delta \Delta F=-\frac{E h}{2}\left[L(w, w)+2 L\left(w, w_{0}\right)\right],
\end{gathered}
$$

where $D=E h^{3} / 12\left(1-v^{2}\right)$ is the flexural rigidity, $\Delta$ stands for the laplacian operator, $c$ accounts for structural damping of the viscous type, $F$ is the Airy stress function, and $L$ is a bilinear operator, whose expression in polar coordinates reads

$$
L(w, F)=w_{, r r}\left(\frac{F_{, r}}{r}+\frac{F_{, \theta \theta}}{r^{2}}\right)+F_{, r r}\left(\frac{w_{, r}}{r}+\frac{w_{, \theta \theta}}{r^{2}}\right)-2\left(\frac{w_{, r \theta}}{r}-\frac{w_{, \theta}}{r^{2}}\right)\left(\frac{F_{, r \theta}}{r}-\frac{F_{, \theta}}{r^{2}}\right) .
$$

The equations are then written with nondimensional variables, by introducing

$$
\begin{gathered}
r=a \bar{r}, \quad t=a^{2} \sqrt{\rho h / D} \bar{t}, \quad w=h \bar{w}, \quad w_{0}=h \bar{w}_{0}, \\
F=E h^{3} \bar{F}, \quad c=\left[E h^{3} / a^{2}\right] \sqrt{\rho h / D} \bar{c} .
\end{gathered}
$$


As nondimensional equations will be used in the remainder of the study, overbars are now omitted in order to write the dimensionless form of the equations of motion

$$
\begin{gathered}
\Delta \Delta w+\ddot{w}=\varepsilon\left[L(w, F)+L\left(w_{0}, F\right)-c \dot{w}\right], \\
\Delta \Delta F=-\frac{1}{2}\left[L(w, w)+2 L\left(w, w_{0}\right)\right]
\end{gathered}
$$

where $\varepsilon=12\left(1-v^{2}\right)$.

The boundary conditions for the case of a free edge write, in nondimensional form [5]

$$
\begin{gathered}
F_{, r}+F_{, \theta \theta}=0, \quad F_{, r \theta}+F_{, \theta}=0, \quad \text { at } r=1, \\
w_{, r r}+v w_{, r}+v w_{, \theta \theta}=0, \quad \text { at } r=1, \\
w_{, r r r}+w_{, r r}-w_{, r}+(2-v) w_{, r \theta \theta}-(3-v) w_{, \theta \theta}=0, \quad \text { at } r=1 .
\end{gathered}
$$

In order to discretize the PDEs, a Galerkin procedure is used. As the eigenmodes cannot be computed analytically because the shape of the imperfection is arbitrary, the eigenmodes of the perfect plate $\Psi_{p}(r, \theta)$ are selected as basis functions. Analytical expressions of $\Psi_{p}(r, \theta)$ involve Bessel functions and can be found in [5]. The unknown displacement is expanded with

$$
w(r, \theta, t)=\sum_{p=1}^{+\infty} q_{p}(t) \Psi_{p}(r, \theta)
$$

where the time functions $q_{p}$ are now the unknowns. In this expression, the subscript $p$ refers to a specific mode of the perfect plate, defined by a couple $(k, n)$, where $k$ is the number of nodal diameters and $n$ the number of nodal circles. If $k \neq 0$, a binary variable is added, indicating the preferential configuration considered (sine or cosine companion mode). Inserting the expansion (2.6) into (2.4a) and (2.4b) and using the orthogonality properties of the expansion functions, the dynamical equations are found to be, for all $p=1 \cdots N$,

$$
\ddot{q}_{p}+2 \xi_{p} \omega_{p} \dot{q}_{p}+\varepsilon\left[\sum_{i=1}^{+\infty} \alpha_{i}^{p} q_{i}+\sum_{i, j=1}^{+\infty} \beta_{i j}^{p} q_{i} q_{j}+\sum_{i, j, k=1}^{+\infty} \Gamma_{i j k}^{p} q_{i} q_{j} q_{k}\right]=0 .
$$

Linear coupling terms between the oscillator equations are present, as the natural modes have not been used for discretizing the PDEs. Analytical expressions of the coupling coefficients $\left(\alpha_{i}^{p}, \beta_{i j}^{p}, \Gamma_{i j k}^{p}\right)$ are given in [42]. The generic viscous damping term $c$ of (2.4a) has been specialized in the discretized equations so as to handle the more general case of a modal viscous damping term of the form $2 \xi_{p} \omega_{p} \dot{q}_{p}$, where $\xi_{p}$ is the damping factor and $\omega_{p}$ the eigenfrequency of mode $p$. On the other hand, external forces have been cancelled, as the remainder of the study will consider free vibrations only.

In order to work with diagonalized linear parts, the matrix of eigenvectors $\mathbf{P}$ of the linear part $\mathbf{L}=\left[\alpha_{i}^{p}\right]_{p, i}$ is numerically computed. A linear change of coordinates is processed, $\mathbf{q}=\mathbf{P X}$, where $\mathbf{X}=\left[X_{1} \cdots X_{N}\right]^{T}$ is, by definition, the vector of modal coordinates, and $N$ is the number of expansion function kept in practical application of the Galerkin's method. Application of 
P makes the linear part diagonal, so that the discretized equations of motion finally writes, $\forall p=1 \cdots N$,

$$
\ddot{X}_{p}+2 \xi_{p} \omega_{p} \dot{X}_{p}+\omega_{p}^{2} X_{p}+\varepsilon\left[\sum_{i, j=1}^{N} g_{i j}^{p} X_{i} X_{j}+\sum_{i, j, k=1}^{N} h_{i j k}^{p} X_{i} X_{j} X_{k}\right]=0
$$

The temporal equations (2.8) describe the dynamics of an imperfect circular plate. The type of nonlinearity can be inferred from these equations. Unfortunately, too severe truncations in (2.8), for example, by keeping only one degree-of-freedom (dof) $(N=1)$ when studying the nonlinear behaviour of the $p$ th mode, lead to incorrect predictions. Nonlinear normal modes (NNMs) offer a clean framework for deriving a single oscillator equation capturing the correct type of nonlinearity [12]. This is recalled in Section 3, where the analytical expression of the coefficient dictating the type of nonlinearity is given.

\subsection{Type of nonlinearity}

Non-linear oscillators differ from linear ones by the frequency dependence on vibration amplitude. The type of nonlinearity defines the behaviour, which can be of the hardening or the softening type.

As shown in [12], NNMs provide an efficient framework for properly truncating nonlinear oscillator equations like (2.8) and predict the type of nonlinearity (hardening or softening behaviour). The method has already been successfully applied to the case of undamped shallow spherical shells in [22]. The main idea is to derive a nonlinear change of coordinates, allowing one to pass from the modal $X_{p}$ coordinates to new-defined normal coordinates $R_{p}$, describing the motions in an invariant-based span of the phase space. The nonlinear change of coordinates is computed from Poincaré and Poincaré-Dulac's theorems, by successive elimination of nonessential coupling terms in the nonlinear oscillator equations. Formally, it reads

$$
\begin{aligned}
X_{p}= & R_{p}+\sum_{i=1}^{N} \sum_{j \geq i}^{N}\left(a_{i j}^{p} R_{i} R_{j}+b_{i j}^{p} S_{i} S_{j}\right)+\sum_{i=1}^{N} \sum_{j=1}^{N} c_{i j}^{p} R_{i} S_{j} \\
& +\sum_{i=1}^{N} \sum_{j \geq i}^{N} \sum_{k \geq j}^{N}\left(r_{i j k}^{p} R_{i} R_{j} R_{k}+s_{i j k}^{p} S_{i} S_{j} S_{k}\right)+\sum_{i=1}^{N} \sum_{j=1}^{N} \sum_{k \geq j}^{N}\left(t_{i j k}^{p} S_{i} R_{j} R_{k}+u_{i j k}^{p} R_{i} S_{j} S_{k}\right), \\
Y_{p}= & S_{p}+\sum_{i=1}^{N} \sum_{j \geq i}^{N}\left(\alpha_{i j}^{p} R_{i} R_{j}+\beta_{i j}^{p} S_{i} S_{j}\right)+\sum_{i=1}^{N} \sum_{j=1}^{N} \gamma_{i j}^{p} R_{i} S_{j} \\
& +\sum_{i=1}^{N} \sum_{j \geq i}^{N} \sum_{k \geq j}^{N}\left(\lambda_{i j k}^{p} R_{i} R_{j} R_{k}+\mu_{i j k}^{p} S_{i} S_{j} S_{k}\right)+\sum_{i=1}^{N} \sum_{j=1}^{N} \sum_{k \geq j}^{N}\left(v_{i j k}^{p} S_{i} R_{j} R_{k}+\zeta_{i j k}^{p} R_{i} S_{j} S_{k}\right) .
\end{aligned}
$$

A third-order approximation of the complete change of coordinates is thus computed. The analytical expressions of the introduced coefficients $\left\{a_{i j}^{p}, b_{i j}^{p}, c_{i j}^{p}, r_{i j k^{\prime}}^{p} s_{i j k^{\prime}}^{p} t_{i j k^{\prime}}^{p} u_{i j k^{\prime}}^{p}\right\}$ and $\left\{\alpha_{i j}^{p}, \beta_{i j}^{p}, \gamma_{i j}^{p}, \lambda_{i j k}^{p}, \mu_{i j k}^{p}, v_{i j k}^{p}, \zeta_{i j k^{\prime}}^{p}\right\}$ are not given here for the sake of brevity. The interested reader may find them in [12] for the undamped case, and in [39] for the damped case. 
Once the nonlinear change of coordinates operated, proper truncations can be realized. In particular, keeping only the normal coordinates $R_{p}$ allows prediction of the correct type of nonlinearity for the $p$ th mode. The dynamics onto the $p$ th NNM reads

$$
\ddot{R}_{p}+\omega_{p}^{2} R_{p}+2 \xi_{p} \omega_{p} \dot{R}_{p}+\left(\varepsilon h_{p p p}^{p}+A_{p p p}^{p}\right) R_{p}^{3}+B_{p p p}^{p} R_{p} \dot{R}_{p}^{2}+C_{p p p}^{p} R_{p}^{2} \dot{R}_{p}=0,
$$

where $A_{p p p}^{p}, B_{p p p}^{p}$, and $C_{p p p}^{p}$ are new coefficients coming from the change of coordinates. Their expressions involve the quadratic coefficients $\left\{g_{i j}^{p}\right\}$ only, together with some of the transformation coefficients, $\left\{a_{i j}^{p}, b_{i j}^{p}, c_{i j}^{p}\right\}$ from (2.9a) and (2.9b) [39]:

$$
\begin{aligned}
& A_{p p p}^{p}=\varepsilon\left[\sum_{l \geq i}^{N} g_{p l}^{p} a_{p p}^{l}+\sum_{l \leq i} g_{l p}^{p} a_{p p}^{l}\right], \\
& B_{p p p}^{p}=\varepsilon\left[\sum_{l \geq i}^{N} g_{p l}^{p} b_{p p}^{l}+\sum_{l \leq i} g_{l p}^{p} b_{p p}^{l}\right], \\
& C_{p p p}^{p}=\varepsilon\left[\sum_{l \geq i}^{N} g_{p l}^{p} c_{p p}^{l}+\sum_{l \leq i} g_{l p}^{p} c_{p p}^{l}\right] .
\end{aligned}
$$

The asymptotic third-order approximation of the dynamics onto the $p$ th NNM given by (2.10) allows one to accurately predict the type of nonlinearity of mode $p$. A first-order perturbative development from (2.10) gives the dependence of the nonlinear oscillation frequency $\omega_{N L}$ on the amplitude of vibration $a$ by the relationship:

$$
\omega_{N L}=\omega_{p}\left(1+T_{p} a^{2}\right)
$$

where $\omega_{p}$ is the natural angular frequency. In this expression, $T_{p}$ is the coefficient governing the type of nonlinearity. If $T_{p}>0$, then hardening behaviour occurs, whereas $T_{p}<0$ implies softening behaviour. The analytical expression for $T_{p}$ writes $[12,22]$

$$
T_{p}=\frac{1}{8 \omega_{p}^{2}}\left[3\left(A_{p p p}^{p}+\varepsilon h_{p p p}^{p}\right)+\omega_{p}^{2} B_{p p p}^{p}\right] .
$$

Finally, the method used for deriving the type of nonlinearity can be summarized as follows. For a geometric imperfection of a given amplitude, the discretization leading to the nonlinear oscillator (2.8) is first computed. The numerical effort associated to this operation is the most important but remains acceptable on a standard computer. Then the nonlinear change of coordinates is computed, which allows derivation of the $A_{p p p}^{p}$ and $B_{p p p}^{p}$ terms occuring in (2.13), the sign of which determines the type of nonlinearity. Numerical results are given in Section 3 for specific imperfections.

\section{Effect of imperfections}

This section is devoted to numerical results about the effect of typical imperfections on the type of nonlinearity of imperfect plates. Two typical imperfections are selected. The first one is axisymmetric and has the shape of mode $(0,1)$, the second one has the shape of the first asymmetric mode $(2,0)$. Consequently, damping is not considered, so that in each equation we have: $\forall p=1 \cdots N, \xi_{p}=0$. The study of the effect of damping will be done separately and is postponed to Section 4 . 


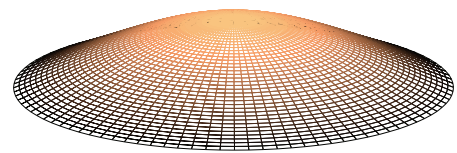

(a)

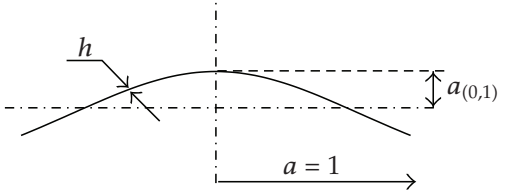

(b)

Figure 2: (a) Three-dimensional view and (b) cross-section of the circular plate with geometric imperfection having the shape of the first axisymmetric mode. As nondimensional quantities are used, $a=1$ and the amplitude $a_{(0,1)}$ of the imperfection is made nondimensional with respect to the thickness $h$.

\subsection{Axisymmetric imperfection}

In this section, the particular case of an axisymmetric imperfection having the shape of mode $(0,1)$ (i.e., with one nodal circle and no nodal diameter) is considered. The expression of the static deflection writes

$$
w_{0}(r)=a_{(0,1)} \Psi_{(0,1)}(r),
$$

where $\Psi_{(0,1)}(r)$ is the mode shape, depending only on the radial coordinate $r$ as a consequence of axisymmetry, and $a_{(0,1)}$ the considered amplitude. The mode shape $\Psi_{(0,1)}(r)$ depends on Bessel function [5], and is shown in Figure 2. The eigenmode is normalized so that $\int_{0}^{1} \Psi_{(0,1)}^{2}(r) d r=1$.

Figure 3 shows the effect of the imperfection on the eigenfrequencies, for an imperfection amplitude from 0 (perfect plate) to $10 \mathrm{~h}$. It is observed that the purely asymmetric modes $(k, 0)$, having no nodal circle and $k$ nodal diameters, are marginally affected by the axisymmetric imperfection. The computation has been done by keeping 51 basis functions: purely asymmetric modes from $(2,0)$ to $(10,0)$, purely axisymmetric modes from $(0,1)$ to $(0,13)$; and mixed modes from $(1,1)$ to $(6,1),(1,2),(2,2),(3,2)$ and $(1,3)$. More details and comparisons with a numerical solution based on finite elements are provided in $[42,43]$. The slight dependence of purely asymmetric eigenfrequencies on an axisymmetric imperfection has already been observed in [44] with the case of the shallow spherical shell.

First, the effect of the imperfection on the axisymmetric modes $(0,1)$ and $(0,2)$ is studied. In this case, the problem is fully axisymmetric so that all the truncations can be limited to axisymmetric modes only, which drastically reduces the numerical burden. The result for mode $(0,1)$ is shown in Figure 4. It is observed that the huge variation of the eigenfrequency with respect to the amplitude of the imperfection results in a quick turn of the behaviour from the hardening to the softening type, occuring for an imperfection amplitude of $a_{(0,1)}=0.38 \mathrm{~h}$. This small value has direct implication for the case of real plates. As the behaviour changes for a fraction of the plate thickness, it should not be intriging to measure a softening behaviour with real plates having small imperfections. This result can also be compared to an earlier result obtained by Hui [10]. Although Hui did not study free-edge boundary condition, he reported a numerical result for the case of simply supported boundary conditions, where the behaviour changes for an imperfection amplitude of $0.28 \mathrm{~h}$. The second main observation inferred from Figure 4 is the occurrence of 2:1 internal resonance between eigenfrequencies, leading to discontinuities in the coefficient $T_{(0,1)}$ dictating the type of nonlinearity. This fact has already been observed and commented for the case of shallow spherical shells in [22]. 


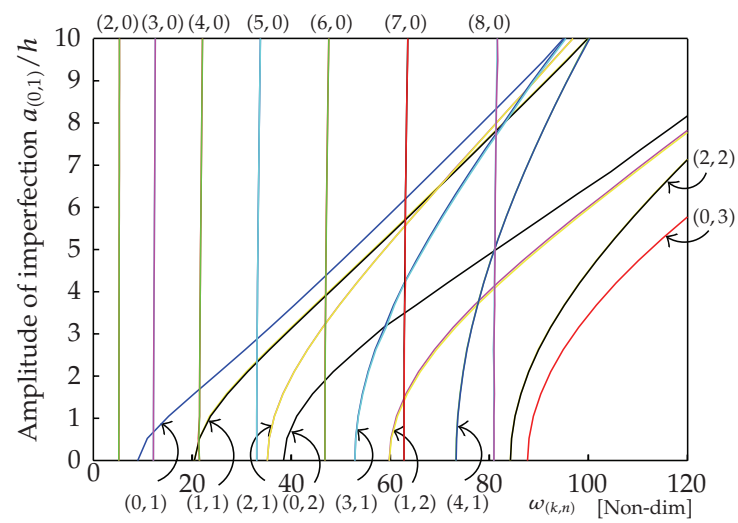

Figure 3: Nondimensional natural frequencies $\omega_{(k, n)}$ of the imperfect plate versus the amplitude of the imperfection having the shape of mode $(0,1)$.

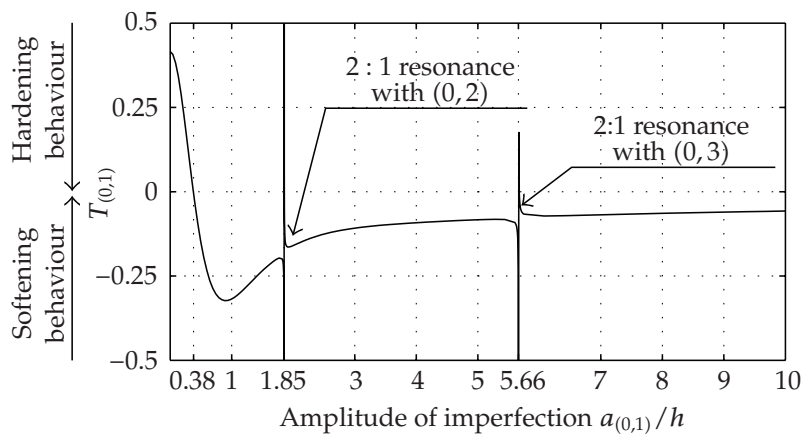

Figure 4: Type of nonlinearity for mode $(0,1)$ with an axisymmetric imperfection having the shape of mode $(0,1)$.

It has also been observed for buckled beams and suspended cables [19, 21]. This is a small denominator effect typical of internal resonance, that is, when the frequency of the studied mode $(0,1)$ exactly fulfills the relationship $2 \omega_{(0,1)}=\omega_{(0, n)}$ with another axisymmetric mode. 2:1 resonance arises here with mode $(0,2)$ at $1.85 \mathrm{~h}$ and with mode $(0,3)$ at $5.66 \mathrm{~h}$. On a practical point of view, one must bear in mind that when 2:1 internal resonance occurs, single-mode solution does not exist anymore, only coupled solutions are possible. Hence the concept of the type of nonlinearity, intimately associated with a single dof behaviour, loses its meaning in a narrow interval around the resonance.

The numerical result for mode $(0,2)$ is shown in Figure 5. Once again, the geometric effect is important and leads to a change of behaviour occurring at $a_{(0,1)}=0.75 \mathrm{~h}$, that is, for a small level of imperfection. 2:1 internal resonance also occurs, thus creating narrow region where hardening behaviour could be observed. This result extends Hui's analysis since only mode $(0,1)$ was studied. Moreover, as a single-mode truncation was used in [10], 2:1 resonances were missed.

Finally, the effect of the imperfection on asymmetric modes is shown in Figure 6 for modes $(2,0)$ and $(4,0)$. The very slight variation of the eigenfrequencies of these modes versus 


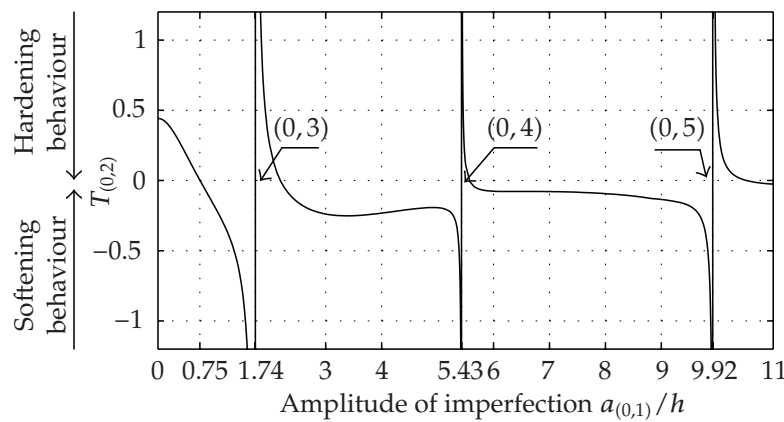

Figure 5: Type of nonlinearity for mode $(0,2)$ with an axisymmetric imperfection having the shape of mode $(0,1)$. 2:1 internal resonances with modes $(0,3),(0,4)$, and $(0,5)$ occurs, respectively, for $a_{(0,1)} / h=1.74,5.43$, and 9.92

the axisymmetric imperfection results in a very slight effect of the geometry. It is observed that before the first 2:1 internal resonance, the type of nonlinearity shows small variations. Hence it is the behaviour of the other eigenfrequencies and the occurrence of 2:1 internal resonance that makes, in these cases, the behaviour turn from hardening to softening behaviour. For mode $(2,0)$, this occurs for an imperfection amplitude of $a_{(0,1)}=0.44 \mathrm{~h}$, where 2:1 resonance with mode $(0,1)$ is observed. For mode $(4,0)$, the first $2: 1$ resonance occurs with mode $(0,2)$ at $a_{(0,1)}=$ $1.39 \mathrm{~h}$, but do not change the behaviour. It is the resonance with mode $(0,1)$ at $a_{(0,1)}=4 \mathrm{~h}$ which makes the behaviour turn from hardening to softening.

These results corroborate those obtained on shallow spherical shells [22]. The fundamental importance of axisymmetric modes in the study of asymmetric ones is confirmed, showing once again that reduction to single mode has no chance to deliver correct results. The behaviour of purely asymmetric modes is found to be of the hardening type until the 2:1 internal resonance with mode $(0,1)$ occurs. However, a specificity of mode $(2,0)$ with regard to all the other purely asymmetric modes is that after this resonance, hardening behaviour (though with a very small value of $\left.T_{(2,0)}\right)$ is observed. This was also the case for shallow spherical shells [22]. Finally, for very large values of the imperfection, the behaviour tends to be neutral.

\subsection{Asymmetric imperfection}

In this section, the effect of an imperfection having the shape of mode $(2,0)$ is studied. Due to the loss of symmetry, degenerated modes are awaited to cease to exist : the equal eigenfrequencies of the sine and cosine configuration of degenerated modes split. In the following, distinction is made systematically between the sine or cosine configuration of companion modes, for example, mode $(2,0, \mathrm{C})$ (resp., $(2,0, \mathrm{~S}))$ refers to the cosine (resp., sine) configuration. More precisely, the imperfection has the shape of $(2,0, C)$ and is shown in Figure 7.

The behaviour of the eigenfrequencies with the imperfection is shown in Figure 8. As expected, the variation of the eigenfrequency corresponding to $(2,0, \mathrm{C})$ is huge, whereas $(2,0, \mathrm{~S})$ keep quite a constant value. The symmetry is not completely broken. One can show that only eigenmodes of the type $(2 k, n)$ split. On the other hand, as shown in Figure 8, modes $(3,0)$, $(5,0)$, and $(1,1)$ are still degenerated. 


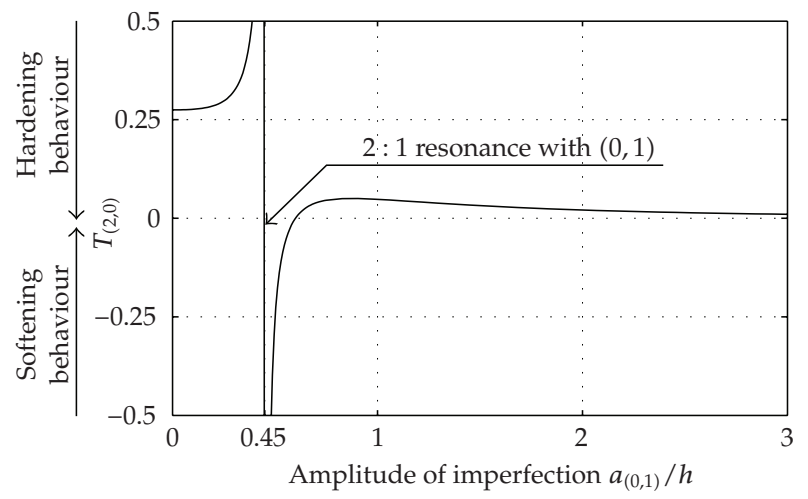

(a)

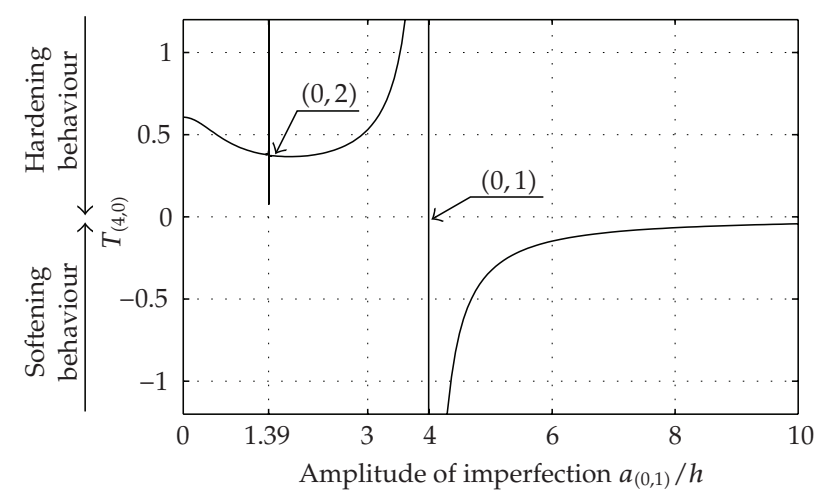

(b)

Figure 6: Type of nonlinearity for $(a)$ : mode $(2,0)$, and $(b)$ : mode $(4,0)$ with an axisymmetric imperfection having the shape of mode $(0,1)$.

The numerical results for type of nonlinearity relative to the two configurations $(2,0, \mathrm{C})$ and $(2,0, \mathrm{~S})$ are shown in Figure 9 . The natural frequency of mode $(2,0, \mathrm{C})$ undergoes a huge variation, which results in a quick change of behaviour, occurring at $0.54 \mathrm{~h}$. Then, a 2:1 internal resonance with $(0,2)$ is noted, but without a noticeable change in the type of nonlinearity, as the interval where the discontinuity present is very narrow. In this case, the behaviour of $T_{(2,0, C)}$ looks like the one observed in the precedent case, that is, the variation of $T_{(0,1)}$ versus an imperfection having the same shape. On the other hand, the eigenfrequency of mode $(2,0, \mathrm{~S})$ remains quite unchanged, so that the behaviour of $T_{(2,0, S)}$ is not much affected by the imperfection until the 2:1 internal resonance is encountered. In that case, the resonance occurs with the other configuration, that is, mode $(2,0, \mathrm{C})$.

Finally, the results for the first two axisymmetric modes $(0,1)$ and $(0,2)$ are shown in Figure 10. Mode $(0,1)$ shows a very slight variation of its eigenfrequency with respect to the asymmetric imperfection $(2,0, \mathrm{C})$. Consequently, the type of nonlinearity is not much affected, until the eigenfrequency of $(2,0, C)$ comes to two times $\omega_{(0,1)}: 2: 1$ internal resonance occurs, and the behaviour becomes softening. On the other hand, the eigenfrequency of $(0,2)$ is more affected by the imperfection. This result in an important decrease of $T_{(0,2)}$ while still remaining positive. A 2:1 internal resonance with $(0,3)$ is encountered for $3.51 \mathrm{~h}$, and two others 2:1 


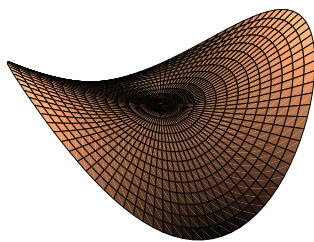

(a)

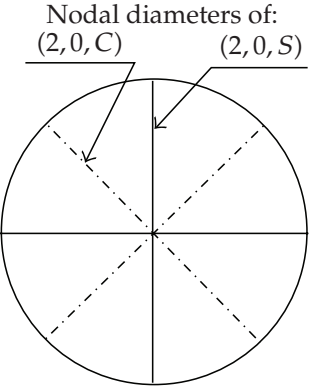

(b)

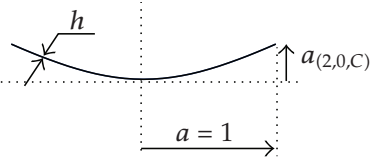

(c)

Figure 7: (a) 3D view, (b) top view, and (c) cross-section along $\theta=0$ for the plate with imperfection having the shape of mode $(2,0, C)$.

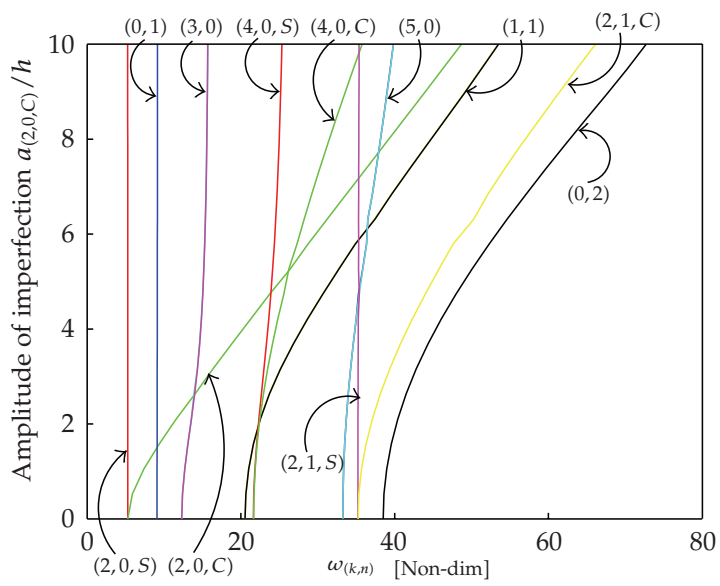

Figure 8: Nondimensional natural frequencies $\omega_{(k, n)}$ of the imperfect plate versus the amplitude of the imperfection having the shape of mode $(2,0, C)$.

resonance, with $(0,4)$ and $(0,5)$, occur around $8 \mathrm{~h}$. However, the interval on which the type of nonlinearity changes its sign is so narrow that it can be neglected. The behaviour is thus mainly of the hardening type for $(0,2)$.

\section{Effect of damping}

In this section, the effect of viscous damping on the type of nonlinearity is addressed. The particular case of the shallow spherical shell is selected to establish the results. The equations of motion are first briefly recalled. Then specific cases of damping are considered, hence complementing the results of [22], that were limited to the undamped shell.

\subsection{The shallow spherical shell equations}

The local equations of motions for the shallow spherical shell can be obtained directly, see [44] for a thorough presentation. They can also be obtained from (2.4a) and (2.4b), by selecting an 


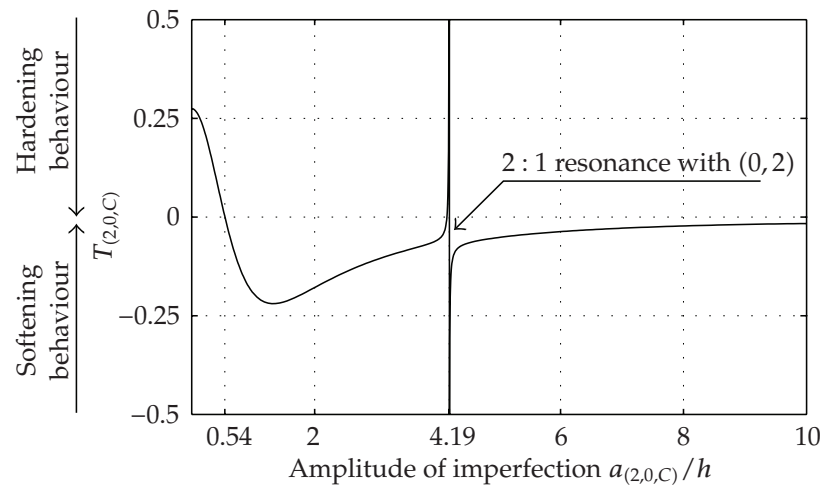

(a)

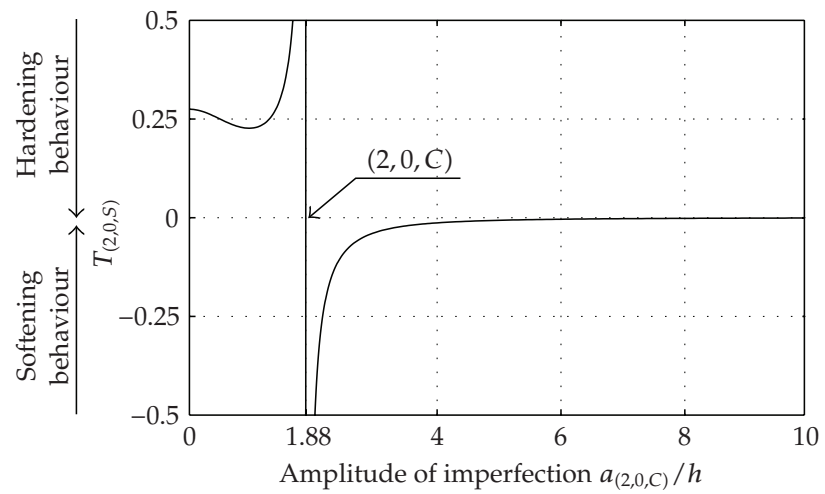

(b)

Figure 9: Type of nonlinearity for $(a)$ : mode $(2,0, C)$ and $(b):(2,0, S)$; for an imperfection having the shape of mode $(2,0, \mathrm{C})$.

imperfection having a spherical shape, as shown in Figure 1(c), see [42]. With $R$, the radius of curvature of the spherical shell ( $R \gg a$ to fulfill the shallow assumption), the local equations write [44]

$$
\begin{gathered}
\Delta \Delta w+\varepsilon_{q} \Delta F+\ddot{w}=\varepsilon[L(w, F)-c \dot{w}+p(r, \theta, t)], \\
\Delta \Delta F-\sqrt{\kappa} \Delta w=-\frac{1}{2} L(w, w),
\end{gathered}
$$

where the aspect ratio $\mathcal{\kappa}$ of the shell has been introduced:

$$
\kappa=\frac{a^{4}}{R^{2} h^{2}},
$$

and $\varepsilon_{q}=12\left(1-v^{2}\right) \sqrt{\kappa}$. The boundary conditions for the case of the spherical shell with free edge write exactly as in the case of the imperfect circular plates so that (2.5a), (2.5b) and (2.5c) are still fulfilled $[42,44]$. A peculiarity of the spherical shell is that all the involved quantities, linear (eigenfrequencies and mode shapes), and nonlinear (coupling coefficients and type of nonlinearity) only depends on $\kappa$, which is the only free-geometric parameter. Hence all the results will be presented as functions of $\kappa$. 


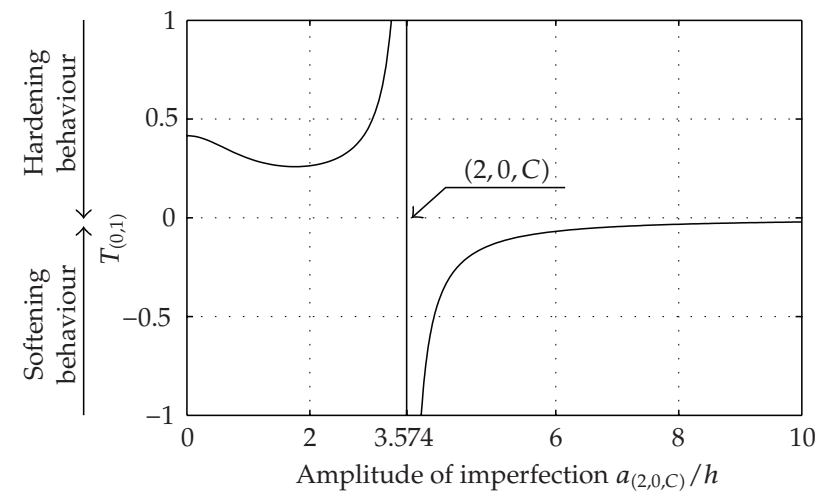

(a)

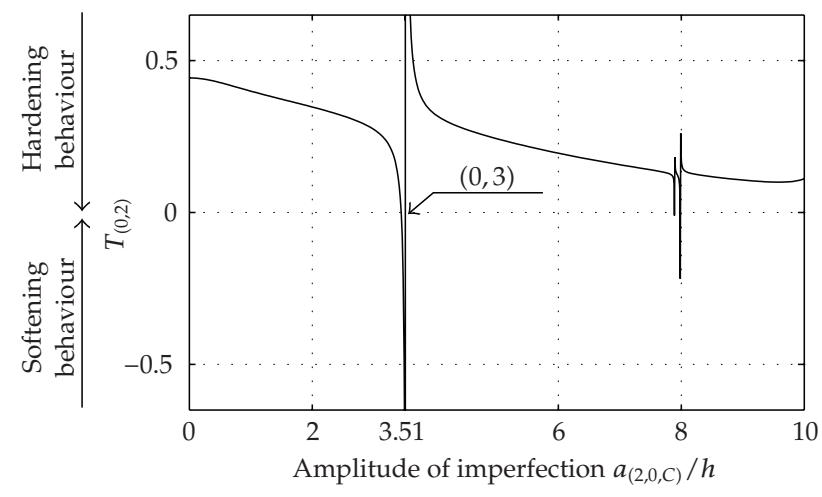

(b)

Figure 10: Type of nonlinearity for $(a)$ : mode $(0,1)$ and $(b):(0,2)$; for an imperfection having the shape of mode $(2,0, C)$.

A Galerkin expansion is used for discretizing the PDEs of motion. As the eigenmodes $\Phi_{p}(r, \theta)$ are known analytically [44], they are used for expanding the unknown transverse displacement:

$$
w(r, \theta, t)=\sum_{p=1}^{+\infty} X_{p}(t) \Phi_{p}(r, \theta)
$$

The modal displacements $X_{p}$ are the unknowns, and their dynamics are governed by, $\forall p \geq 1$

$$
\ddot{X}_{p}+2 \xi_{p} \omega_{p} \dot{X}_{p}+\omega_{p}^{2} X_{p}+\varepsilon_{q} \sum_{i, j=1}^{+\infty} \widetilde{g}_{i j}^{p} X_{i} X_{j}+\varepsilon \sum_{i, j, k=1}^{+\infty} \widetilde{h}_{i j k}^{p} X_{i} X_{j} X_{k}=0 .
$$

The analytical expressions for the quadratic and cubic coupling coefficients $\left(\widetilde{g}_{i j}^{p} \widetilde{h}_{i j k}^{p}\right)$ involve integrals of products of eigenmodes on the surface, they can be found in [22, 44]. As in Section 3, a modal viscous damping term of the form $2 \xi_{p} \omega_{p} \dot{X}_{p}$ is considered, whereas external forces have been cancelled as only free responses are studied. 


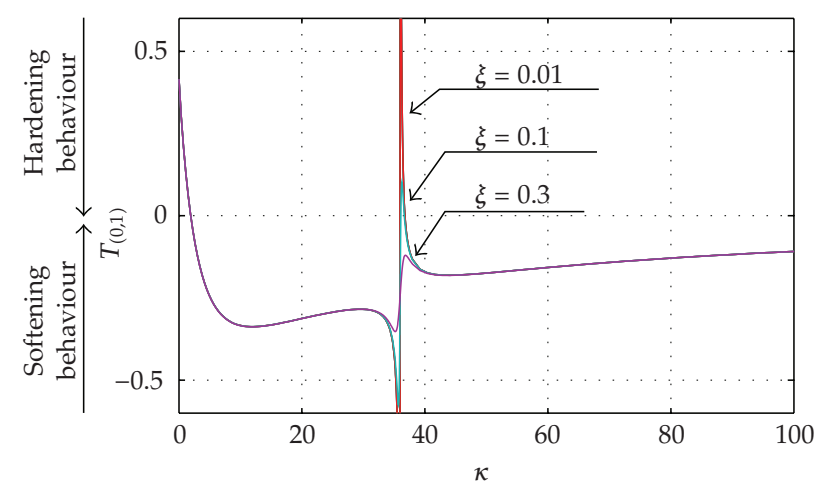

(a)

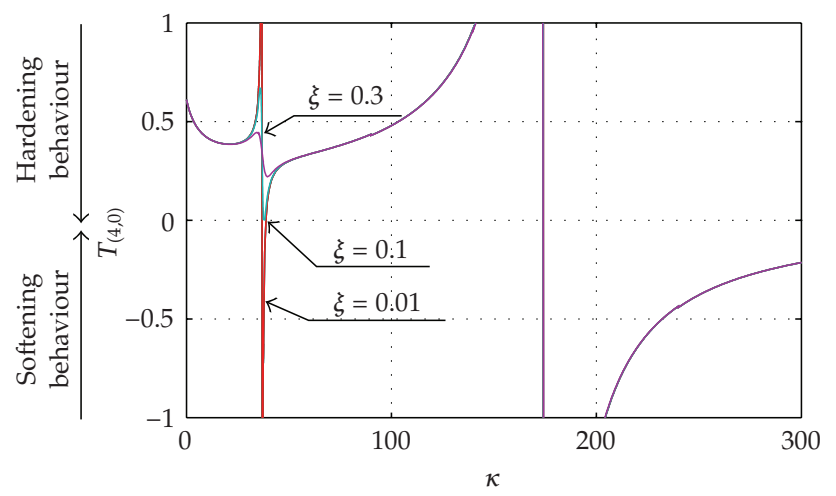

(b)

Figure 11: Type of nonlinearity for $(a)$ : mode $(0,1)$ and $(b):(4,0)$ versus the aspect ratio $\kappa$ of a shallow spherical shell. Increasing values of damping for Case $1\left(\forall p=1 \cdots N, \xi_{p}=\xi / \omega_{p}\right)$ are shown, with $\xi=0$ and 0.01 (red), 0.1 (cyan) and 0.3 (violet).

The type of nonlinearity can be inferred from (4.4) by using the NNM method. The results for an undamped shell have already been computed and are presented in [22]. However, an extension of the NNM-method, taking into account the damping term, has been proposed in [39]. Amongst other things, it has been shown on a simple two dofs system of coupled oscillators, that the type of nonlinearity depends on the damping. The aim of this section is thus to complement the results presented in [22] for documenting the dependence of a shell on viscous damping and for assessing its effect.

\subsection{Numerical results}

Three cases are selected in order to derive results for a variety of damping behaviours:

Case 1. For all $p=1 \cdots N, \xi_{p}=\xi / \omega_{p}$;

Case 2. For all $p=1 \cdots N, \xi_{p}=\xi$;

Case 3. For all $p=1 \cdots N, \xi_{p}=\xi \omega_{p}$; 


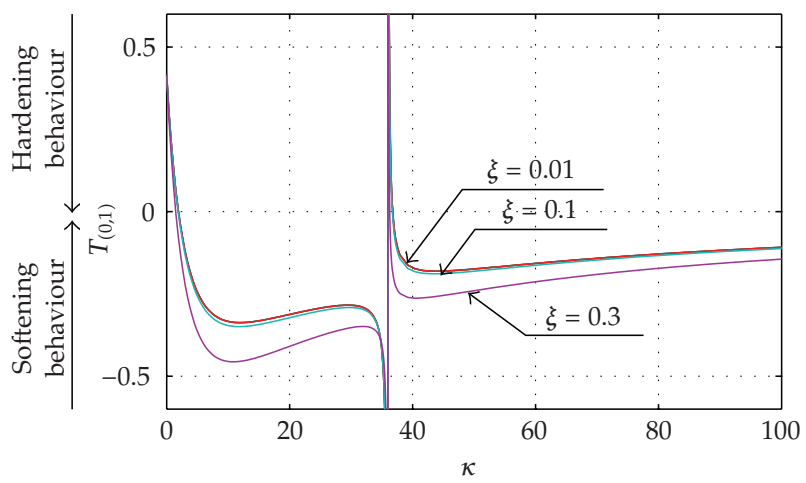

(a)

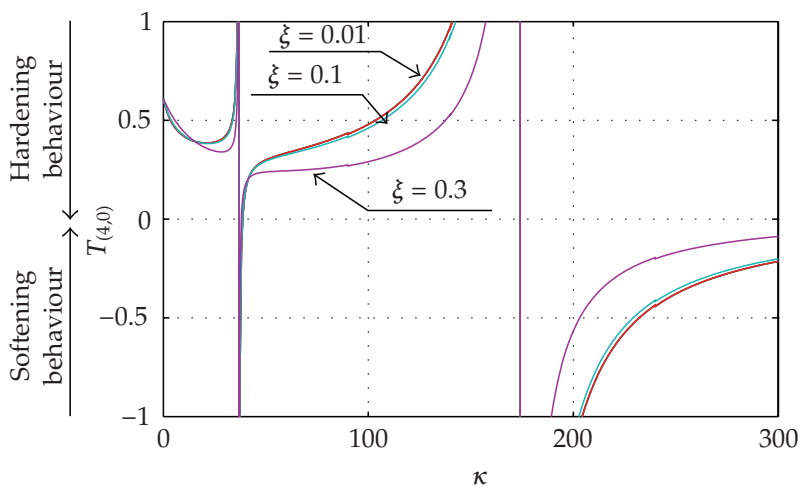

(b)

Figure 12: Type of nonlinearity for $(\mathrm{a})$ : mode $(0,1)$ and $(\mathrm{b}):(4,0)$ versus the aspect ratio $\kappa$. Increasing values of damping for Case $2\left(\forall p=1 \cdots N, \xi_{p}=\xi\right)$ are shown, with $\xi=0$ and 0.01 (red), 0.1 (cyan), and 0.3 (violet).

In the above cases, $\xi$ is a constant value, ranging from 0 to 0.3 . Case 1 corresponds to a decay factor $\left(2 \xi_{p} \omega_{p}=2 \xi\right)$ that is independent from the frequency, that is, with a constant $2 \xi$ value for any mode. With a small value of $\xi$, it may model the low-frequency (i.e., below the critical frequency) behaviour of thin metallic structures such as aluminium plates $[45,46]$. Case 2 describes a decay factor that is linear with the frequency, and may model, for instance, damped structures as glass plates in the low-frequency range [45]. Finally, Case 3 accounts for a strongly damped structure, with a center manifold limited to a few modes.

The effect of increasing damping is shown for modes $(0,1)$ and $(4,0)$, for Case 1 in Figure 11, Case 2 in Figure 12, and Case 3 in Figure 13. Mode $(0,1)$ undergoes a rapid change of behaviour: the transition from hardening to softening type nonlinearity occurs at $\kappa=1.93$. Then 2:1 internal resonance with mode $(0,2)$ occurs at $\mathcal{\kappa}=36$, but the behaviour remains of the softening type. Mode $(4,0)$ displays a hardening behaviour until the 2:1 resonance with mode $(0,1)$ at $\kappa=174.1$. The first resonance with $(0,2)$ at $\mathcal{\kappa}=36.9$ does not change the behaviour on a large interval. Adding the damping of Case 1 shows that the discontinuity ocurring at 2:1 internal resonance is smoothened. However, it happens for a quite large amount of damping in the structure. Damping values of $0,1 \mathrm{e}-4,1 \mathrm{e}-3$, and $1 \mathrm{e}-2$ have been tested and 


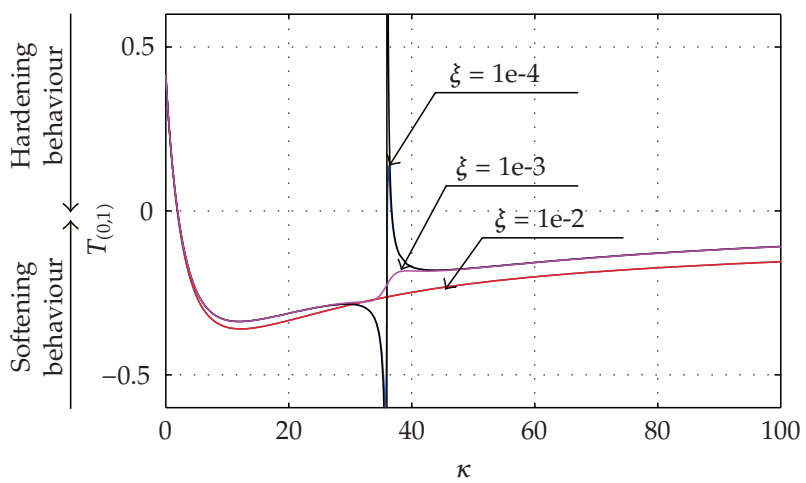

(a)

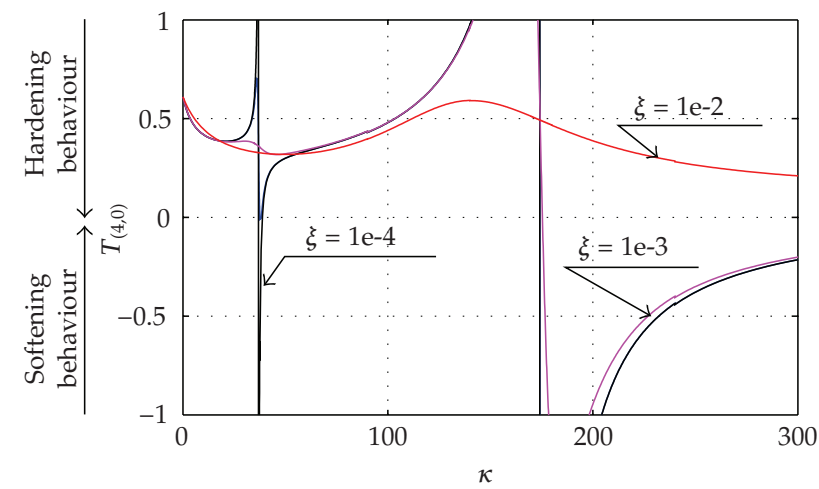

(b)

Figure 13: Type of nonlinearity for $(a)$ : mode $(0,1)$ and $(b):(4,0)$ versus the aspect ratio $\kappa$. Increasing values of damping for Case $3\left(\forall p=1 \cdots N, \xi_{p}=\xi \omega_{p}\right)$ are shown, with $\xi=0$ and $1 \mathrm{e}-4$ (black), $1 \mathrm{e}-3$ (magenta), and $1 \mathrm{e}-2$ (red).

give exactly the same behaviour so that only one curve is visible in Figure 11. Large values of the damping term $\xi$, namely, 0.1 and 0.3 (which correspond to strongly damped structures) must be selected to see the discontinuity smoothened. Moreover, outside the narrow intervals where 2:1 resonance occurs, the effect of damping is not visible. As a conclusion for Case 1, it appears that this kind of damping has a really marginal effect on the type of nonlinearity, so that undamped results can be estimated as reliable for lightly damped structures with modal damping factor below 0.1 .

Case 2 corresponds to a more damped structure than Case 1 . However, it is observed in Figure 12 that the discontinuity is not smoothened at the 2:1 internal resonance. Inspecting back the analytical results shows that this is a natural consequence of the expression of the coefficients of the nonlinear change of coordinates for asymptotic NNMs. When the specific Case of constant damping factors is selected, small denominators remain present. On the other hand, outside the regions of 2:1 resonance, the effect of damping is pronounced and enhances the softening behaviour. But once again, very large values of damping factors such as 0.3 must be reached to see a prominent influence.

Finally, Case 3 depicts the case of a rapidly increasing decay factor with respect to the frequency. As the overall damping in the structure is thus larger, smaller values of $\xi$ have been 
selected, namely, 1e-4, 1e-3, and 1e-2. $\xi=1 \mathrm{e}-4$ gives quite coincident results with $\xi=0$. But from $\xi=1 \mathrm{e}-3$, the effect of the damping is very important: the discontinuities are smoothened, except the larger one occurring for mode $(4,0)$ with mode $(0,1)$. For $\xi=1 \mathrm{e}-2,2: 1$ resonance are not visible anymore. A particular result with this value is for mode $(4,0)$ : the smoothening effect is so important that the nonlinearity remains of the hardening type. Finally, the fact that the damping generally favours the softening behaviour cannot be declared as a general rule, as one counterexample has been exhibited here. From these results, it can be inferred that the damping has little incidence on the type of nonlinearity for thin structures, until very large values are attained. It is observed that the damping generally favours the softening behaviour, but this rule is not true in general. In particular when the transition from hardening to softening type nonlinearity is due to a 2:1 internal resonance and is not the direct effect of the change of geometry, a large value of damping may favours hardening behaviour, as observed here for mode $(4,0)$ in Case 3.

\section{Conclusion}

The effect of geometric imperfections on the hardening/softening behaviour of circular plates with a free edge has been studied. Thanks to the NNMs, quantitative results for the transition from hardening to softening behaviour has been documented, for a number of modes and for two typical imperfections. Two general rules have been observed from the numerical results: for modes which eigenfrequency strongly depends on the imperfection, the type of nonlinearity changes rapidly, and softening behaviour occurs for a very small imperfection with an amplitude being a fraction of the plate thickness. On the other hand, some eigenfrequencies show a slight dependence with the considered imperfection. For these, 2:1 internal resonances are the main factor for changing the type of nonlinearity. In a second part of the paper, the effect of viscous damping on the type of nonlinearity of shallow spherical shells has been studied. It has been shown quantitatively that this effect is slight for usual damping values encountered in thin structures.

\section{References}

[1] S. A. Tobias, "Free undamped nonlinear vibrations of imperfect circular disks," Proceedings of the Institution of Mechanical Engineers, vol. 171, pp. 691-715, 1957.

[2] N. Yamaki, "Influence of large amplitudes on flexural vibrations of elastic plates," Zeitschrift für Angewandte Mathematik und Mechanik, vol. 41, no. 12, pp. 501-510, 1961.

[3] K. A. V. Pandalai and M. Sathyamoorthy, "On the modal equations of large amplitude flexural vibration of beams, plates, rings and shells," International Journal of Non-Linear Mechanics, vol. 8, no. 3, pp. 213-218, 1973.

[4] S. Sridhar, D. T. Mook, and A. H. Nayfeh, "Nonlinear resonances in the forced responses of plates-I: symmetric responses of circular plates," Journal of Sound and Vibration, vol. 41, no. 3, pp. 359-373, 1975.

[5] C. Touzé, O. Thomas, and A. Chaigne, "Asymmetric nonlinear forced vibrations of free-edge circular plates-I: theory," Journal of Sound and Vibration, vol. 258, no. 4, pp. 649-676, 2002.

[6] O. Thomas, C. Touzé, and A. Chaigne, "Asymmetric nonlinear forced vibrations of free-edge circular plates-II: experiments," Journal of Sound and Vibration, vol. 265, no. 5, pp. 1075-1101, 2003.

[7] P. L. Grossman, B. Koplik, and Y.-Y. Yu, "Nonlinear vibrations of shallow spherical shells," Journal of Applied Mechanics, vol. 36, no. 3, pp. 451-458, 1969.

[8] D. Hui, "Larg-amplitude vibrations of geometrically imperfect shallow spherical shells with structural damping," AIAA Journal, vol. 21, no. 12, pp. 1736-1741, 1983.

[9] K. Yasuda and G. Kushida, "Nonlinear forced oscillations of a shallow spherical shell," Bulletin of the Japan Society of Mechanical Engineers, vol. 27, no. 232, pp. 2233-2240, 1984. 
[10] D. Hui, "Large-amplitude axisymmetric vibrations of geometrically imperfect circular plates," Journal of Sound and Vibration, vol. 91, no. 2, pp. 239-246, 1983.

[11] A. H. Nayfeh, J. F. Nayfeh, and D. T. Mook, “On methods for continuous systems with quadratic and cubic nonlinearities," Nonlinear Dynamics, vol. 3, no. 2, pp. 145-162, 1992.

[12] C. Touzé, O. Thomas, and A. Chaigne, "Hardening/softening behaviour in nonlinear oscillations of structural systems using nonlinear normal modes," Journal of Sound and Vibration, vol. 273, no. 1-2, pp. 77-101, 2004.

[13] M. Amabili, F. Pellicano, and M. P. Païdoussis, "Nonlinear vibrations of simply supported, circular cylindrical shells, coupled to quiescent fluid," Journal of Fluids and Structures, vol. 12, no. 7, pp. 883918, 1998.

[14] E. H. Dowell, "Comments on the nonlinear vibrations of cylindrical shells," Journal of Fluids and Structures, vol. 12, no. 8, pp. 1087-1089, 1998.

[15] M. Amabili, F. Pellicano, and M. P. Païdoussi, "Further comments on nonlinear vibrations of shells," Journal of Fluids and Structures, vol. 13, no. 1, pp. 159-160, 1999.

[16] D. A. Evensen, "Nonlinear vibrations of cylindrical shells-logical rationale," Journal of Fluids and Structures, vol. 13, no. 1, pp. 161-164, 1999.

[17] M. Amabili and M. P. Païdoussis, "Review of studies on geometrically nonlinear vibrations and dynamics of circular cylindrical shells and panels, with and without fluid-structure interaction," Applied Mechanics Reviews, vol. 56, no. 4, pp. 349-356, 2003.

[18] M. Amabili, Nonlinear Vibrations and Stability of Shells and Plates, Cambridge University Press, New York, NY, USA, 2008.

[19] G. Rega, W. Lacarbonara, and A. H. Nayfeh, "Reduction methods for nonlinear vibrations of spatially continuous systems with initial curvature," in IUTAM Symposium on Recent Developments in Nonlinear Oscillations of Mechanical Systems (Hanoi, 1999), vol. 77 of Solid Mech. Appl., pp. 235-246, Kluwer Academic Publishers, Dordrecht, The Netherlands, 2000.

[20] F. Pellicano, M. Amabili, and M. P. Païdoussis, "Effect of the geometry on the nonlinear vibration of circular cylindrical shells," International Journal of Non-Linear Mechanics, vol. 37, no. 7, pp. 1181-1198, 2002.

[21] H. N. Arafat and A. H. Nayfeh, "Nonlinear responses of suspended cables to primary resonance excitations," Journal of Sound and Vibration, vol. 266, no. 2, pp. 325-354, 2003.

[22] C. Touzé and O. Thomas, "Nonlinear behaviour of free-edge shallow spherical shells: effect of the geometry," International Journal of Non-Linear Mechanics, vol. 41, no. 5, pp. 678-692, 2006.

[23] A. Rosen and J. Singer, "Effect of axisymmetric imperfections on the vibrations of cylindrical shells under axial compression," AIAA Journal, vol. 12, no. 7, pp. 995-997, 1974.

[24] D. Hui and A. W. Leissa, "Effects of uni-directional geometric imperfections on vibrations of pressurized shallow spherical shells," International Journal of Non-Linear Mechanics, vol. 18, no. 4, pp. 279-285, 1983.

[25] P. B. Goncalves, "Axisymmetric vibrations of imperfect shallow spherical caps under pressure loading," Journal of Sound and Vibration, vol. 174, no. 2, pp. 249-260, 1994.

[26] M. Amabili, "A comparison of shell theories for large-amplitude vibrations of circular cylindrical shells: Lagrangian approach," Journal of Sound and Vibration, vol. 264, no. 5, pp. 1091-1125, 2003.

[27] V. D. Kubenko and P. S. Koval'chuk, "Influence of initial geometric imperfections on the vibrations and dynamic stability of elastic shells," International Applied Mechanics, vol. 40, no. 8, pp. 847-877, 2004.

[28] E. L. Jansen, "The effect of geometric imperfections on the vibrations of anisotropic cylindrical shells," Thin-Walled Structures, vol. 45, no. 3, pp. 274-282, 2007.

[29] C.-Y. Chia, "Nonlinear free vibration and postbuckling of symmetrically laminated orthotropic imperfect shallow cylindrical panels with two adjacent edges simply supported and the other edges clamped," International Journal of Solids and Structures, vol. 23, no. 8, pp. 1123-1132, 1987.

[30] D. Hui and A. W. Leissa, "Effects of geometric imperfections on vibrations of biaxially compressed rectangular flat plates," Journal of Applied Mechanics, vol. 50, no. 4, pp. 750-756, 1983.

[31] N. Yamaki, K. Otomo, and M. Chiba, "Nonlinear vibrations of a clamped circular plate with initial deflection and initial edge displacement-I: theory," Journal of Sound and Vibration, vol. 79, no. 1, pp. 23-42, 1981. 
[32] N. Yamaki, K. Otomo, and M. Chiba, "Nonlinear vibrations of a clamped circular plate with initial deflection and initial edge displacement-II: experiment," Journal of Sound and Vibration, vol. 79, no. 1, pp. 43-59, 1981.

[33] N. Yamaki and M. Chiba, "Nonlinear vibrations of a clamped rectangular plate with initial deflection and initial edge displacement-I: theory," Thin-Walled Structures, vol. 1, no. 1, pp. 3-29, 1983.

[34] N. Yamaki, K. Otomo, and M. Chiba, "Nonlinear vibrations of a clamped rectangular plate with initial deflection and initial edge displacement-I: experiment," Thin-Walled Structures, vol. 1, no. 1, pp. 101119, 1983.

[35] M. Amabili, "Theory and experiments for large-amplitude vibrations of rectangular plates with geometric imperfections," Journal of Sound and Vibration, vol. 291, no. 3-5, pp. 539-565, 2006.

[36] M. Amabili, "Theory and experiments for large-amplitude vibrations of empty and fluid-filled circular cylindrical shells with imperfections," Journal of Sound and Vibration, vol. 262, no. 4, pp. 921-975, 2003.

[37] M. Amabili, "Theory and experiments for large-amplitude vibrations of circular cylindrical panels with geometric imperfections," Journal of Sound and Vibration, vol. 298, no. 1-2, pp. 43-72, 2006.

[38] C. C. Lin and L. W. Chen, "Large-amplitude vibration of an initially imperfect moderatly thick plate," Journal of Sound and Vibration, vol. 135, no. 2, pp. 213-224, 1989.

[39] C. Touzé and M. Amabili, "Nonlinear normal modes for damped geometrically nonlinear systems: application to reduced-order modelling of harmonically forced structures," Journal of Sound and Vibration, vol. 298, no. 4-5, pp. 958-981, 2006.

[40] G. J. Efstathiades, "A new approach to the large-deflection vibrations of imperfect circular disks using Galerkin's procedure," Journal of Sound and Vibration, vol. 16, no. 2, pp. 231-253, 1971.

[41] G. L. Ostiguy and S. Sassi, "Effects of initial geometric imperfections on dynamic behaviour of rectangular plates," Nonlinear Dynamics, vol. 3, no. 3, pp. 165-181, 1992.

[42] C. Camier, C. Touzé, and O. Thomas, "Nonlinear vibrations of imperfect free-edge circular plates," submitted to European Journal of Mechanics: A/Solids .

[43] C. Camier, C. Touzé, and O. Thomas, "Effet des imperfections géométriques sur les vibrations nonlinéaires de plaques circulaires minces," in Proceedings of 18 ème Congrès Français de Mécanique, Grenoble, France, August 2007.

[44] O. Thomas, C. Touzé, and A. Chaigne, "Nonlinear vibrations of free-edge thin spherical shells: modal interaction rules and 1:1:2 internal resonance," International Journal of Solids and Structures, vol. 42, no. 11-12, pp. 3339-3373, 2005.

[45] A. Chaigne and C. Lambourg, "Time-domain simulation of damped impacted plates-I: theory and experiments," Journal of the Acoustical Society of America, vol. 109, no. 4, pp. 1422-1432, 2001.

[46] M. Amabili, "Nonlinear vibrations of rectangular plates with different boundary conditions: theory and experiments," Computers and Structures, vol. 82, no. 31-32, pp. 2587-2605, 2004. 


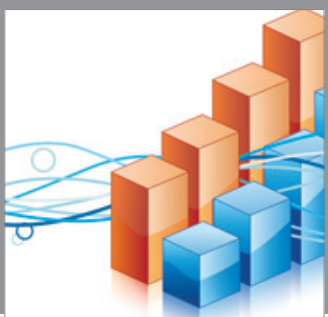

Advances in

Operations Research

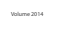

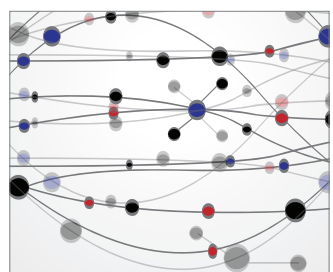

\section{The Scientific} World Journal
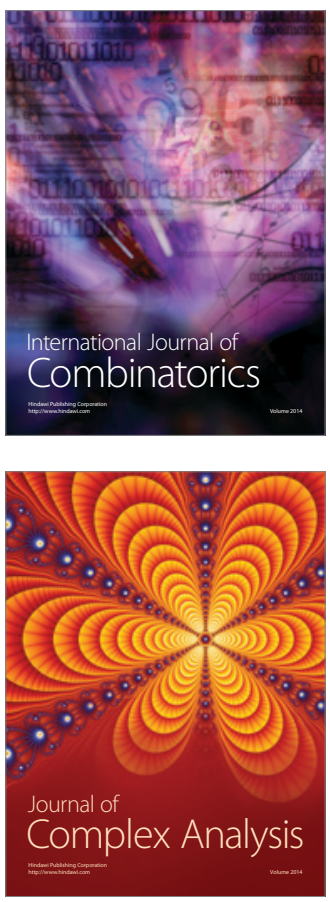

International Journal of

Mathematics and

Mathematical

Sciences
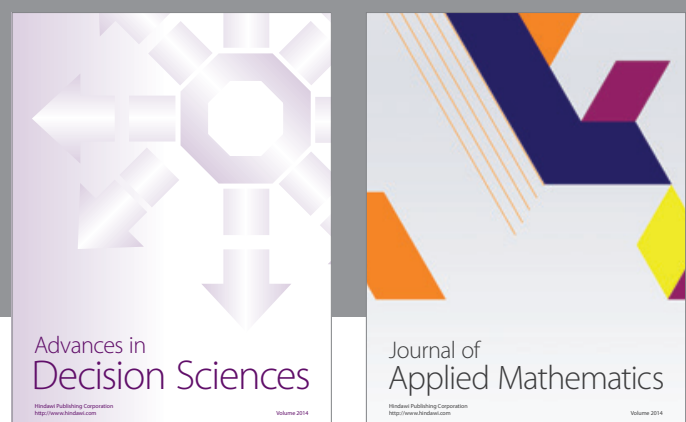

Journal of

Applied Mathematics
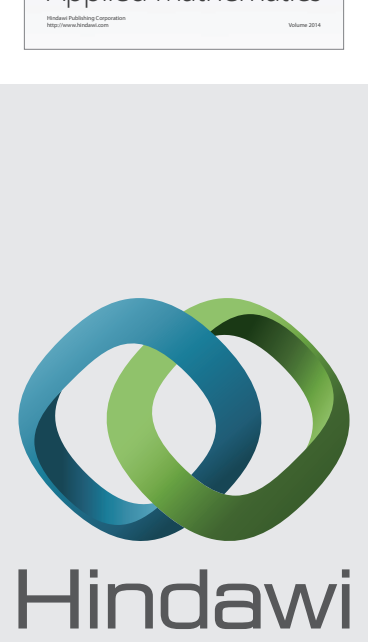

Submit your manuscripts at http://www.hindawi.com
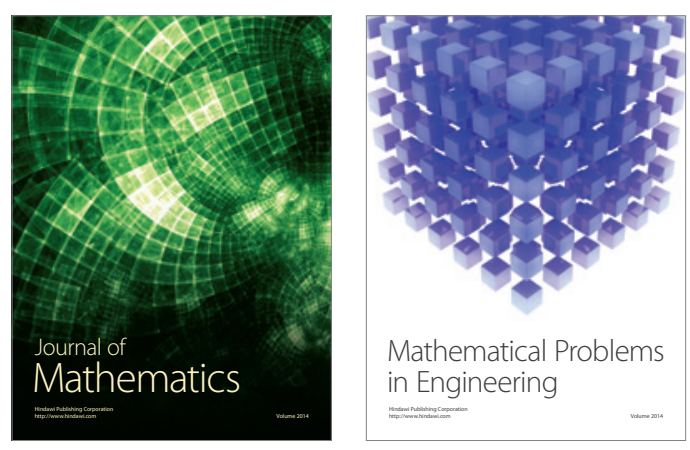

Mathematical Problems in Engineering
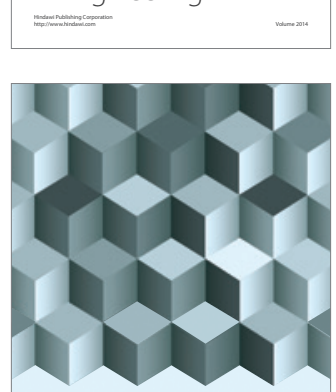

Journal of

Function Spaces
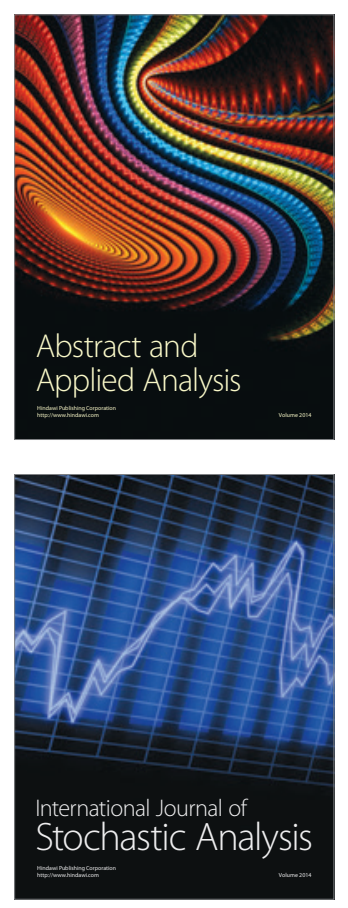

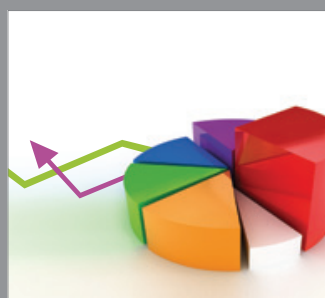

ournal of

Probability and Statistics

Promensencen
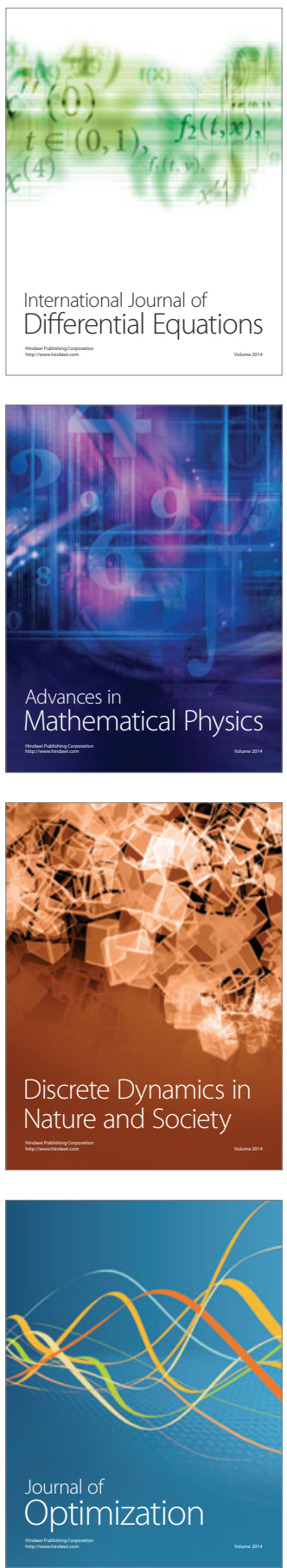